\title{
Neutron Irradiation Effects on Mechanical and Magnetic Properties of Pre-deformed Iron-based Model Alloys
}

\author{
Y. Kamada, J. N. Mohapatra, H. Kikuchi, S. Kobayashi, T. Murakami, and H. Watanabe* \\ Faculty of Engineering, Iwate Univ., 4-3-5 Ueda, Morioka 020-8551, Japan \\ *Research Institute for Applied Mechanics, Kyushu Univ., Kasuga 816-8580, Japan
}

\begin{abstract}
The purpose of this study was to clarify the role of pre-existing dislocations on the mechanical and magnetic properties of neutron-irradiated steels. Magnetic domain observation of electron-irradiated well-annealed iron revealed that irradiation defects actually disturbed domain wall movement. Pre-deformed pure iron and iron-based alloys were neutron-irradiated, and their hardness and magnetic hysteresis loops were measured. Decreasing behaviors in coercivity suggest the domain walls moved more easily after irradiation. Also, the role of pre-existing dislocation is discussed by taking into consideration the change in hardness.
\end{abstract}

Key words: irradiation embrittlement, lattice defect, coercivity, nondestructive evaluation

\section{Introduction}

The irradiation embrittlement of reactor pressure vessel (RPV) steels in ageing nuclear power plants is a serious issue from the viewpoint of safety throughout the world. There is a need to develop suitable non-destructive evaluation (NDE) techniques to diagnose degradation. As RPV steels are iron based alloys, magnetic measurement is a promising method for evaluation. Mechanical and magnetic hardening are expected to simultaneously occur due to the formation of lattice defects because defects generally obstruct dislocation and magnetic domain wall movements, ${ }^{1,2}$. In-situ magnetic hysteresis measurements during neutron irradiation revealed two kinds of trends in coercivity change ${ }^{3)}$. Monotonically increasing behaviour was observed in coercivity in well-annealed pure iron. In contrast, that of RPV steel decreased at high neutron fluence. RPV steels have bainitic structures that contain high density dislocations. Such pre-existing dislocations behave as sinks for irradiation defects, and these defects tend to form near pre-existing dislocations ${ }^{4}$, which have various effects on magnetic properties. Therefore, the different trends in coercivity between pure iron and RPV steel may result from the different densities of pre-existing dislocations.

Two experiments were conducted in this study. First, well-annealed pure iron with low dislocation density was electron-irradiated and magnetic domain wall movement was observed. Second, pre-deformed iron-based alloys with high dislocation density were neutron-irradiated and their magnetic properties were investigated. The role of pre-existing dislocations on the magnetic properties of neutron-irradiated RPV steels is discussed by taking these results into consideration.

\section{Experimental Procedures}

\subsection{Electron-irradiated pure iron}

A piece of pure-iron was rolled to obtain a foil, and then punched into disks $3 \mathrm{~mm}$ in diameter. The disks were well-annealed at $1073 \mathrm{~K}$ to remove the effects of rolling and to reduce the dislocation density. A hole and an extremely thin region around the hole were prepared at the center of the disk by conventional twin-jet polishing. Then, the specimen was installed into the stage of a high voltage electron microscope (JEM-1000) of $1.0 \mathrm{MeV}$ at Kyushu University, and irradiated with electrons. The irradiation temperature was $473 \mathrm{~K}$ and the total damage was $0.15 \mathrm{dpa}$ (displacement per atom). The electron-irradiated specimens are not radioactive and can therefore be examined outside. The magnetic domain wall was observed by Lorentz transmission electron microscopy (Philips Tecnai ${ }^{\mathrm{TM}}$ ) at Iwate University.

\subsection{Neutron-irradiated pre-deformed iron and} iron-based alloys

Pure iron and $\mathrm{Fe}-\mathrm{X}$ alloys $(\mathrm{X}=0.6 \% \mathrm{Mn}, 0.4 \% \mathrm{Si}$, and $1 \% \mathrm{Cu}$ ) were cold-rolled to introduce a large number of dislocations. The compositions of $\mathrm{Mn}$ and $\mathrm{Si}$ were similar to those of RPV steels. Although the typical composition of $\mathrm{Cu}$ in $\mathrm{RPV}$ steels is less than $0.1 \%$, this specimen was over-doped to accelerate $\mathrm{Cu}$ precipitation, which is the origin of irradiation embrittlement when steels contain copper as an impurity. The $\mathrm{Fe}-\mathrm{Cu}$ alloy was quenched from $1073 \mathrm{~K}$ before the cold rolling. Therefore, $\mathrm{Cu}$ atoms were distributed randomly in a super-saturated solid solution state. The ratios of cold-rolling reduction in thickness, $\left(t_{\text {initial }}-t_{\text {rolled }}\right) / t_{\text {initial }} \times$ 100, are summarized in Table 1. It was difficult to evaluate the exact ratio for $\mathrm{Fe}-\mathrm{Mn}$ alloy due to its thinness, but it was estimated to be more than $70 \%$. Plate-shaped specimens $(l=20, w=6, t=0.2$ to $0.4 \mathrm{~mm})$ were cut from the rolled plates after rolling.

Neutron irradiation was carried out in the Belgian test reactor BR2 up to fluencies of $1.0 \times 10^{24} \mathrm{n} / \mathrm{m}^{2}$ at 573 $\mathrm{K}$ (commercial operating temperature). Since the specimens become radioactive, $B-H$ hysteresis loops and micro-Vickers hardness measurements were performed 
at the hot laboratory of the International Research Center for Nuclear Materials Science, Institute for Materials Research (IMR), at Tohoku University. The setup for the magnetic measurement system is illustrated in Fig. 1. The plate specimens were inserted into exciting and detecting coils. A triangular current of $2 \mathrm{~Hz}$ was applied through a bipolar source, and the signals were collected with a personal computer through an amplifier, filter, and analog-to-digital converter. The $B-H$ hysteresis loops were obtained using LabVIEW software.

\section{Results and Discussion}

\subsection{Domain wall observation of electron-irradiated pure} iron

First, the relation between the magnetic domain wall and irradiation defects formed in pure iron with low dislocation density was investigated. A focused image of electron-irradiated pure iron is shown in Fig. 2. Many irradiation defects can clearly be seen in areas where the high-energy electron beam was exposed. Some of them would be interstitial-type dislocation loops, which are similar to the lattice defects observed in neutron-irradiated pure iron. When the image is defocused, a magnetic domain wall can be observed due to deflection of the electron beam by Lorentz forces. In our system, the magnetic field along the beam axis can be controlled during the domain observation. Since the specimen was slightly tilted to obtain a clear image, the magnetic field of an in-plane component appeared, which can be used to move the domain wall of thin specimens.

Discontinuous domain wall movements were confirmed during the continuous change in applied magnetic field. Figure 3 shows a typical snap-shot where the domain wall stopped. The observed area is almost the same as that in Fig. 2. By comparing the two images, we were able to confirm that the domain walls stopped along the irradiation defects (arrows in Fig. 2). This means that the irradiation defects pinned the movement of the domain walls. In this case, the defects were a few hundreds of nanometers. Such defects were also found in the neutron-irradiated pure iron specimen $^{3)}$. Therefore, the origin of the increase of coercivity observed during neutron irradiation of pure iron $^{3)}$ resulted from the domain wall pinning by the irradiation defects.

\subsection{Hardness of neutron-irradiated pre-deformed iron and iron-based alloys}

Next, the effect of pre-existing dislocation was investigated using pre-deformed specimens. Figure 4 summarizes the Vickers hardness of four pre-deformed specimens before and after neutron irradiation. Although the hardness of pure iron decreased slightly, those of the other specimens increased.
Table 1 Conditions of cold rolling.

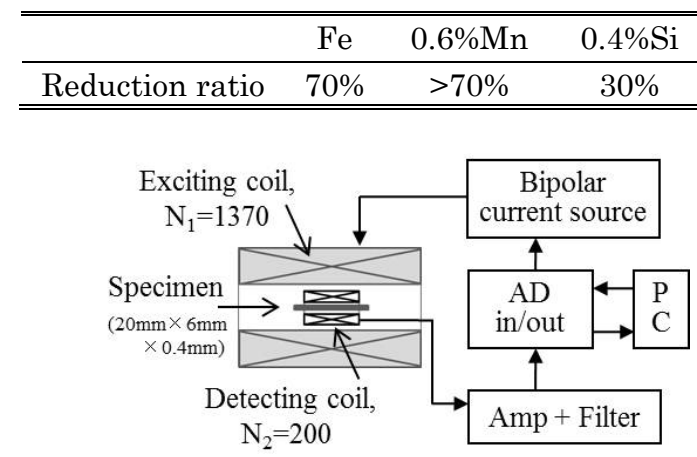

Fig. 1 Setup for magnetic measurements.

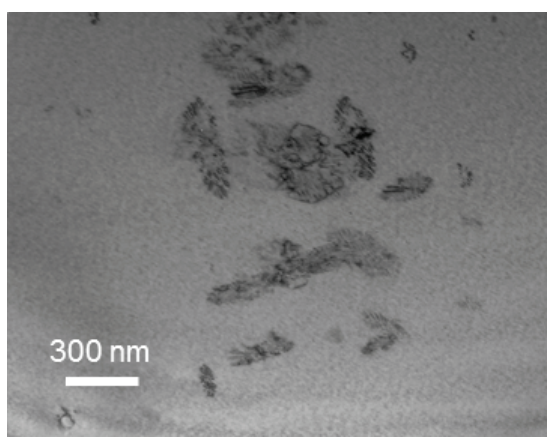

Fig. 2 Focused image of electron-irradiated iron.

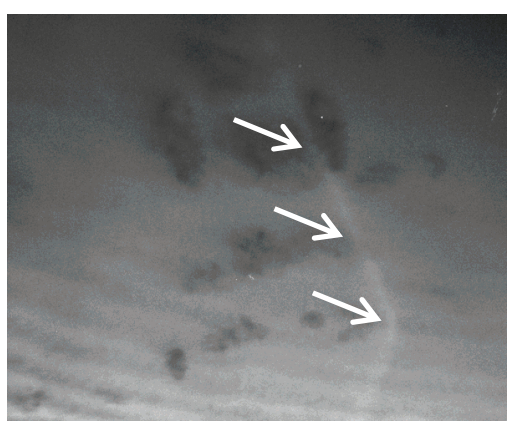

Fig. 3 Defocused image of electron-irradiated iron.

The thermal relaxation of pre-existing dislocation microstructures may have occurred at this irradiation temperature of $573 \mathrm{~K}$, which caused the reduction in hardness. The balance between the formation of irradiation defects that caused an increase in hardness, and the relaxation of pre-existing dislocation microstructures determined the trend in hardness changes. The iron-based alloys contained a second element that possibly enhanced the nucleation of irradiation defect clusters. Therefore, the hardness of $\mathrm{Fe}-\mathrm{Mn}, \mathrm{Fe}-\mathrm{Si}$, and $\mathrm{Fe}-\mathrm{Cu}$ alloys shows increasing behaviors. In particular, the hardness of the $\mathrm{Fe}-1 \% \mathrm{Cu}$ alloy increased greatly due to the formation of $\mathrm{Cu}$ rich precipitates. 


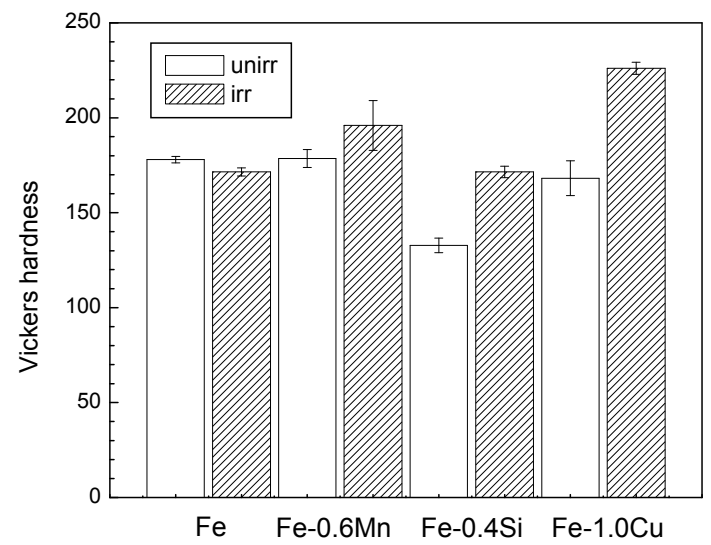

Fig. 4 Vickers hardness of pre-deformed specimens before and after irradiation.

\subsection{Magnetic properties of neutron-irradiated pre-deformed iron and iron-based alloys}

Figure 5 plots the $B-H$ loops of pre-deformed $\mathrm{Fe}-0.6 \% \mathrm{Mn}$ alloy before and after irradiation. We used a solenoid coil to apply a magnetic field. Therefore, the magnetization curves inclined due to the demagnetization effect. Figure 5(b) has magnifications of the $B-H$ loops around the coercivity. Fig. 5 (b) clearly shows that the coercivity of $\mathrm{Fe}-\mathrm{Mn}$ alloy decreases after irradiation. Since the coercivity is not influenced by the demagnetization effect, our discussion is focused on changes in coercivity.

The coercivity of other specimens was also evaluated from each $B$ - $H$ loop. They are summarized in Fig. 6. Coercivity decreased due to neutron irradiation in all specimens. An especially large reduction was confirmed for specimens made of pure iron and $\mathrm{Fe}-0.6 \% \mathrm{Mn}$ alloy. Both were heavily pre-deformed, and hence, their dislocation densities were larger. The fact the specimens with large dislocation densities had large reductions in coercivity suggests that pre-existing dislocation play an important role in determining the effects irradiation has on magnetic properties.

It is notable that the hardness of the three alloys increased but their coercivity decreased due to irradiation, as can be seen in Figs. 4 and 6. Hardness decreased slightly for pure iron, but its coercivity greatly decreased. These results indicate that the hardness is sensitive to the formation of irradiation defects, but that the coercivity strongly reflects the change in the state of pre-existing dislocation microstructures. Two plausible mechanisms for the reduction in coercivity can be described as follows. First, the relaxation of dislocation microstructures due to thermal rearrangement releases internal stress. Such changes in internal stress make domain walls move more easily; hence, the coercivity decreases. Second, there is a possibility that the formation of irradiation defects reduce the internal stress around pre-existing dislocations. Irradiation defects easily form near dislocations due to the elastic strain fields around dislocations $^{4)}$. In this case, the strain field of pre-existing dislocations would be compensated for by that of irradiation defects, and hence, the coercivity decreases.
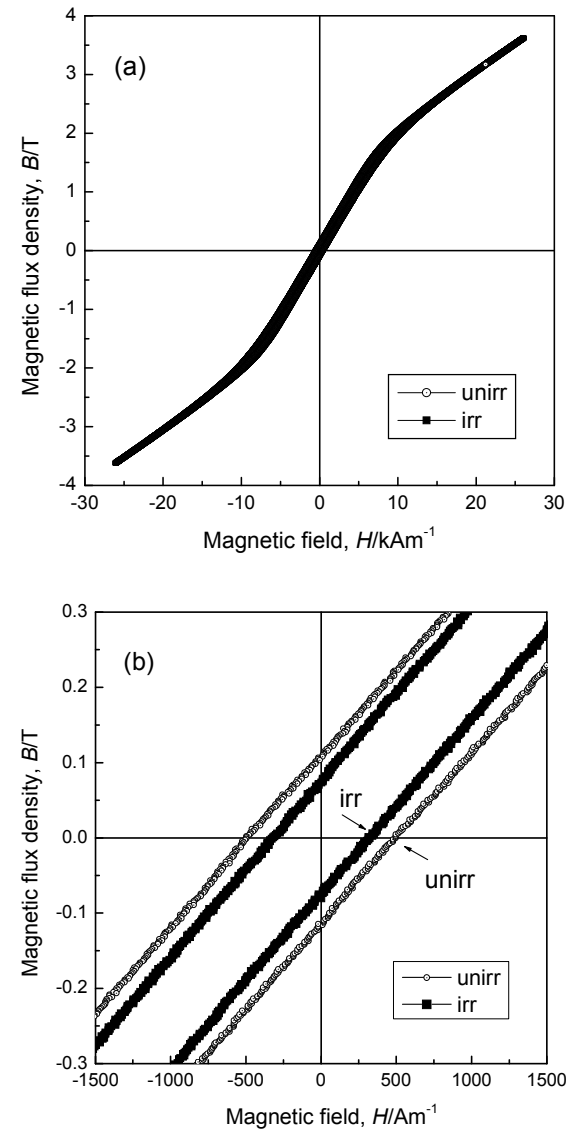

Fig. $5 \quad B$ - $H$ loops of pre-deformed Fe-0.6\%Mn before and after irradiation; (b) is a magnification of (a).

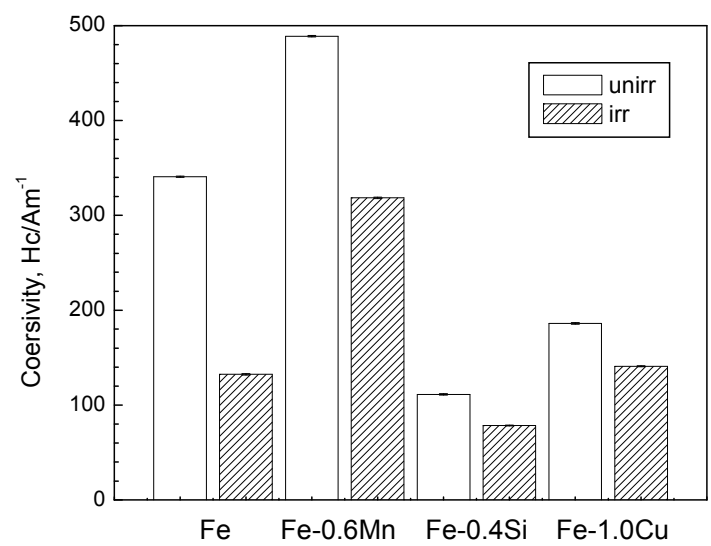

Fig. 6 Coercivity of pre-deformed specimens before and after irradiation. 


\subsection{Role of pre-existing dislocations}

As we explained in Subsection 3.1, irradiation defects actually hinder the domain wall movement, which causes an increase in coercivity. This supports the previous results obtained from in-situ magnetic measurements of well-annealed iron during neutron irradiation $^{3)}$. However, the role of pre-existing dislocations on magnetic properties becomes also important in pre-deformed pure iron and iron-based alloys with high dislocation densities. The coercivity of the pre-deformed specimens decreased because of irradiation, which is a similar trend observed in RPV steel at high neutron fluence ${ }^{3)}$. Since actual RPV steel contains a large number of dislocations, a careful interpretation is required to develop magnetic NDE methods for irradiation embrittlement.

\section{Summary}

Magnetic domain walls were observed in electron-irradiated iron, and the hindrance of domain walls due to irradiation defects was confirmed. However, a decrease in coercivity was found in neutron-irradiated pre-deformed iron and iron-based alloys, which could have been caused by the reduced internal stress in pre-existing dislocations due to relaxation and compensation effects. This study suggested that it is necessary to consider the role of pre-existing dislocations in addition to that of irradiation defects, when we discuss the effect of neutron irradiation on the mechanical and magnetic properties of RPV steels with high dislocation density.

Acknowledgements This work was supported in part by a Grant-in-Aid for JSPS Fellows, KAKENHI (22.00390), and a Grant-in-Aid for Scientific Research (B), No. 23360418, from the Ministry of Education, Culture, Sports, Science and Technology of Japan. This work was carried out under a collaborative research program by the Research Institute for Applied Mechanics at Kyushu University, and the International Research Center for Nuclear Materials Science, Institute for Materials Research (IMR), at Tohoku University.

\section{References}

1) H. Kronmuller and M. Fahnle: Micromagnetism and the microstructure of ferromagnetic solids, p71 (Cambridge University Press, UK, 2003).

2) H. Trauble: Magnetism and metallurgy, Berkowitz AE, Kneller E (eds), p 621 (Academic Press, New York, 1969).

3) S. Takahashi, H. Kikuchi, K. Ara, N. Ebine, Y. Kamada, S. Kobayashi, and M. Suzuki: J. Appl. Phys., 100, 023902 (2006).

4) N. M. Ghoniem, B. N. Singh, L. Z. Sun, T. Diaz de la Rubia: J. Nucl. Mater., 276, 166-177 (2000).

Received October 19, 2012

Accepted December 13, 2012 